\title{
VISUALISATION DES RÉSEAUX DE VORTEX PAR DÉCORATION AVEC DES PARTICULES MAGNÉTIQUES (*)
}

\author{
B. LETELLIER
}

Laboratoires de Marcoussis, CRCGE, 91460 Marcoussis, France

\begin{abstract}
Résumé. - Après avoir rappelé le principe des dispositifs utilisés jusqu'à ce jour pour visualiser les réseaux de vortex des supraconducteurs de deuxième espèce par décoration de la surface avec des particules magnétiques, nous analysons les processus qui interviennent pendant la décoration. Cette analyse nous permet de mettre en évidence les paramètres importants et de proposer une nouvelle technique. Les premiers résultats expérimentaux obtenus avec celle-ci sont présentés.

Abstract. - After recalling the principle of the apparatus hitherto used to visualize flux-line
lattices in type II superconductors by decoration of the specimen surface with magnetic particles,
we analyse the processes occurring during the decoration. This analyse allows us to suggest a new
decoration technique. First experimental results obtained by using this technique are presented.
\end{abstract}

1. Introduction. - La situation actuelle montre que les théories et les moyens expérimentaux habituellement utilisés sont insuffisants pour comprendre les mécanismes qui contrôlent l'ancrage et le mouvement des vortex dans les supraconducteurs de deuxième espèce. Les théories existantes comportent encore beaucoup d'arbitraire et le contexte expérimental est assez confus du fait des informations très indirectes que fournissent les mesures les plus habituellement utilisées : densités de courant critique, aimantation.

Parallèlement à la poursuite d'études systématiques sur l'ancrage dans des matériaux de structure métallique connue, il est nécessaire de s'intéresser aux propriétés du réseau de vortex. Pour ce faire, trois voies sont actuellement possibles : la résonance magnétique nucléaire, la diffraction de neutrons et la visualisation par décoration avec des particules magnétiques. Les deux premières sont décrites, au cours de ce colloque, respectivement par J. M. Delrieu et par P. Thorel et R. Kahn.

La visualisation la plus séduisante et la plus riche d'enseignements serait certainement une visualisation dynamique puisqu'elle permettrait de révéler les mouvements des vortex. Cependant elle présente de telles difficultés que toutes les tentatives ont échoué jusqu'à présent. On est donc ramené à une photographie du réseau à un instant donné qui est possible grâce à une méthode mise au point initialement par Traüble et Essmann à Stuttgart et par Sarma et Moon à Newcastle.

Cette méthode est analogue, dans son principe, à celle de Bitter [1] pour révéler les parois de domaines ferromagnétiques. Elle consiste à évaporer du fer dans une atmosphère d'hélium gazeux sous une pres-

(*) Cette étude a été soutenue par la DRME. sion de l'ordre de 0,1 torr. Le libre parcours moyen des atomes évaporés est alors suffisamment faible, de l'ordre de $10^{-2} \mathrm{~cm}$, pour que les chocs avec les atomes d'hélium entraînent une nucléation de particules. Ces particules se thermalisent dans le gaz et sont transportées vers la surface de l'échantillon où, en s'accumulant aux points d'émergence des vortex, elles révèlent le réseau de vortex au voisinage de la surface de l'échantillon. Un examen au microscope électronique, sur une réplique de la surface, permet d'observer le spectre magnétique ainsi obtenu.

La technique utilisée par Traüble et Essmann consiste à évaporer le fer dans de l'hélium gazeux à une pression de 0,8 torr en équilibre avec un bain à $1,23 \mathrm{~K}$. L'échantillon est fixé sur un support en cuivre qui trempe dans l'hélium liquide [2], [3], [4], [5]. La technique utilisée par Sarma et Moon consiste à évaporer le fer dans une enceinte plongée dans le bain d'hélium liquide d'un cryostat. L'échantillon est fixé sur le fond du récipient [6], [7], [8], [9], [10]. Nous avions initialement adopté une technique comparable à celle de Sarma et Moon (Fig. 1) grâce à laquelle nous avons pu observer des réseaux de vortex sur différents échantillons (Fig. 2).

Ces techniques ont permis l'observation de réseaux de vortex correspondant à des inductions inférieures ou égales à 2000 gauss avec une probabilité de réussite (nombre d'essais réussis/nombre total d'essais) de l'ordre de $10 \%$. Elles ont apporté un grand nombre de renseignements concernant les réseaux de vortex : nature des défauts de réseau, symétries corrélées avec l'orientation cristalline de l'échantillon, intéraction attractive entre vortex dans les supraconducteurs de deuxième espèce à faible paramètre de GinsburgLandau, ...

Bien que la mise en œuvre de ces observations ne 


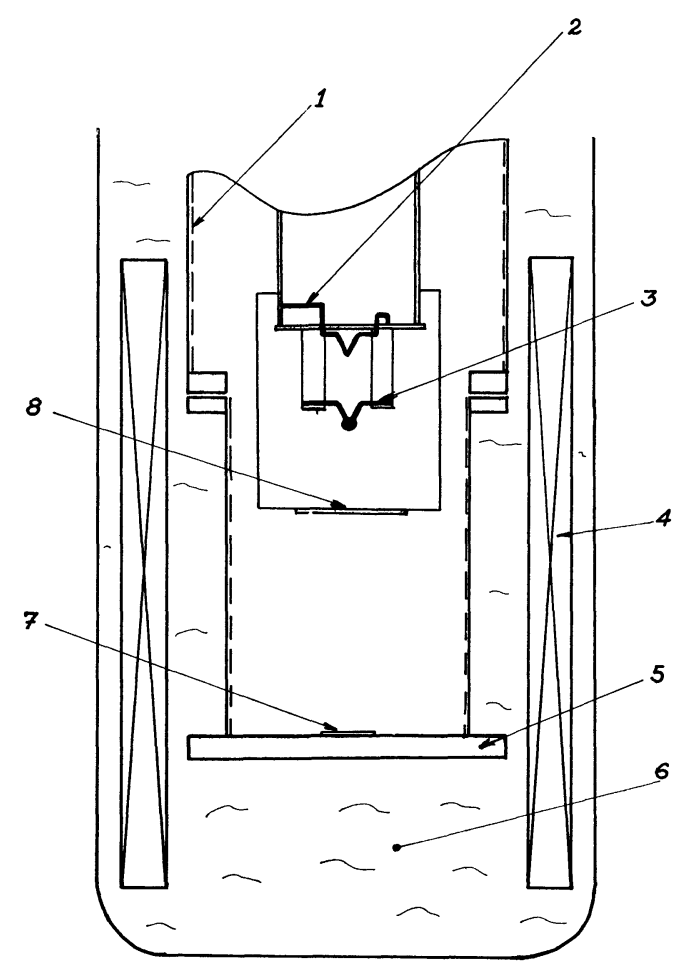

Fig. 1. - Chambre d'évaporation utilisée précédemment : 1) paroi noircie ; 2) filament de dégazage (on le chauffe avant l'évaporation) ; 3) filament d'évaporation du fer ; 4) bobine supraconductrice; 5) fond en cuivre; 6) bain d'hélium du cryostat ; 7) échantillon ; 8) écran anti-rayonnement.

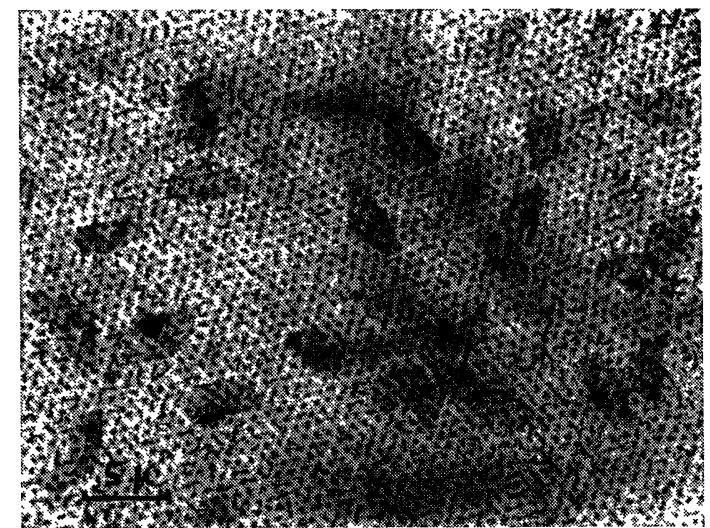

(a)

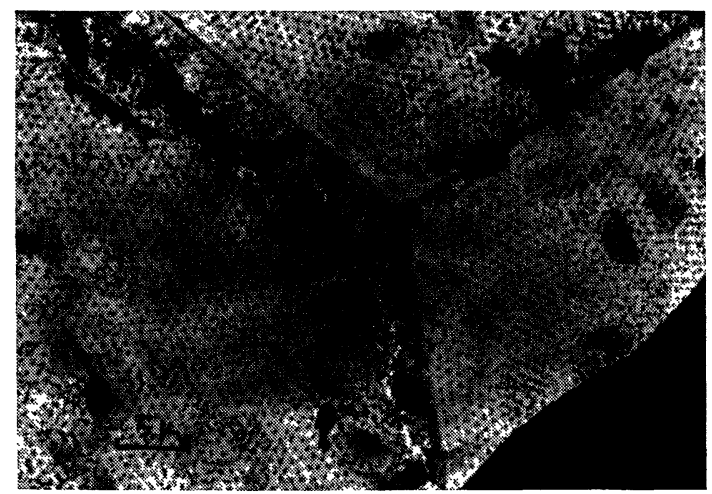

(b)

Fig. 2a. - Distribution du flux dans une plaquette de plomb $4 \%$ at indium, champ appliqué : 200 Oe $2 b$; réseau de vortex à un joint de grain du métal. demande que des moyens expérimentaux relativement modestes, comparés à ceux nécessaires à la diffraction de neutrons ou à la résonance magnétique nucléaire, la faible probabilité de réussite de cette méthode décourage beaucoup d'équipes intéressées par celle-ci. Afin de pouvoir utiliser plus systématiquement ce moyen d'investigation, il convient d'en accroître la probabilité de réussite et d'augmenter l'induction à laquelle il est possible d'effectuer des visualisations. C'est dans ce but que nous nous sommes efforcé de comprendre et de contrôler les processus qui interviennent pendant la décoration. Dans les paragraphes ci-dessous, après avoir analysé ces processus, nous discutons les performances des dispositifs existants puis nous proposons une nouvelle technique et nous rapportons les premiers résultats expérimentaux obtenus avec celle-ci.

2. Analyse des processus qui interviennent pendant la décoration. - Pour qu'une particule aille se poser au cœur d'un vortex, il faut que son gain d'énergie potentielle, dû à la modulation du champ magnétique au voisinage de la surface de l'échantillon, soit supérieur ou égal à son énergie cinétique. L'induction maximale, $B_{\max }$,que peuvent révéler les particules de masse $M$, de moment magnétique $\mu$ et de vitesse $v_{0}$ est donc donnée par la relation :

$$
\delta H\left(B_{\max } ; x\right) \gtrsim \frac{M v_{0}^{2}}{2 \mu}
$$

où $\delta H\left(B_{\max } ; x\right)$ est la modulation de champ magnétique à une distance $x$ de la surface de l'échantillon comparable à l'encombrement des particules.

Cette relation montre que les paramètres qui déterminent la valeur de $B_{\max }$ sont la taille et la nature des particules et leur vitesse au voisinage de la surface de l'échantillon.

La taille des particules intervient dans l'expression de la modulation du champ magnétique $\delta H(B ; x)$. Cette modulation de champ, qui peut être calculée à partir de la modulation du champ magnétique à l'intérieur de l'échantillon [11] en utilisant la méthode due à $\mathrm{P}$. F. Milleron et al. [12], est une fonction décroissante de $B$ et de $x$. La figure 3 représente $\delta H(B ; x)$ pour un supraconducteur ayant des paramètres $K$ et $H_{\mathrm{C} 2}$ voisins de ceux des échantillons sur lesquels ont été effectuées des décorations jusqu'à maintenant.

La nature des particules intervient par la valeur du rapport $M / \mu$. Ce rapport peut être estimé en utilisant les résultats des travaux de A. Tasaki et al. [13] qui ont mesuré le moment magnétique de particules de diamètre compris entre 80 et $2000 \AA$. Ainsi pour des particules de fer de dimensions comparables à celles utilisées pour les décorations, $300 \AA$, le moment magnétique est égal à un magnéton de Bohr par atome.

La vitesse $V_{0}$ des particules au voisinage de la surface de l'échantillon est déterminée par les mécanismes 


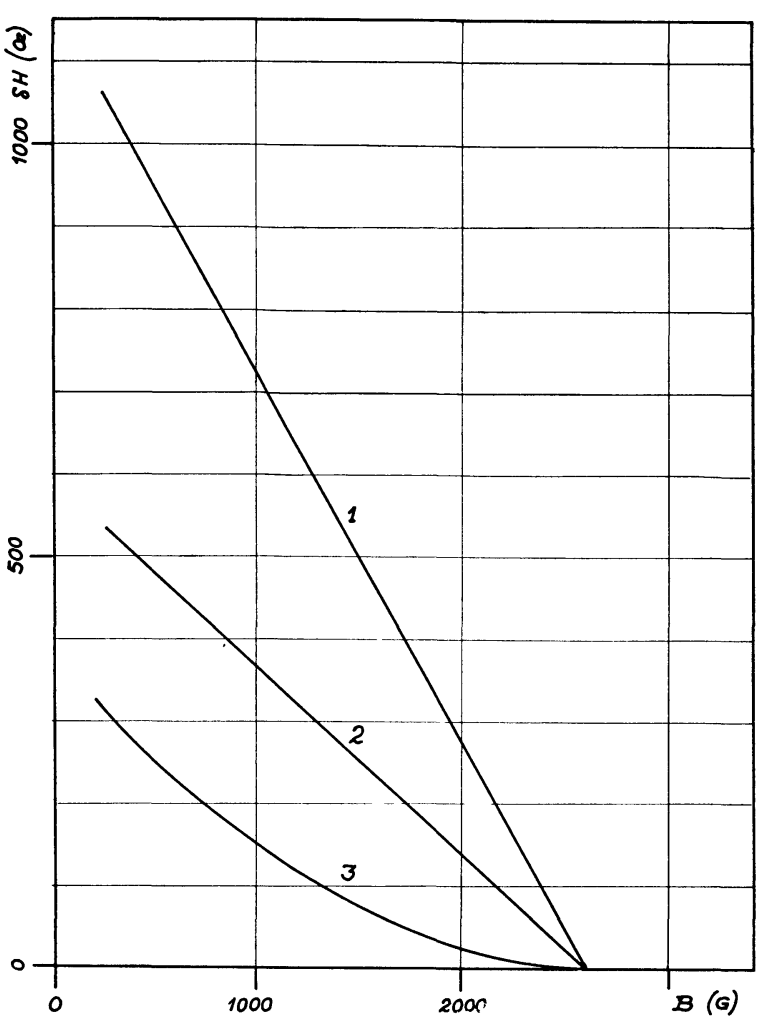

Fig. 3. - Modulation du champ magnétique : 1) dans ; 2) à la surface ; 3) à $200 \AA$ de la surface d'un supraconducteur tel que

$$
K=1 \text { et } H_{\mathrm{C}_{2}}=2600 \mathrm{Oe} \text {. }
$$

assurant le transport des particules entre la zone où elles sont nucléées et la surface de l'échantillon. Une détermination exacte de la valeur de cette vitesse est relativement délicate du fait de la complexité des processus mis en jeu, mais il est possible d'en estimer l'ordre de grandeur en adoptant une description simplifiée.

Loin de la surface de l'échantillon, les particules sont soumises à des champs de force : pesanteur, gradients de champ magnétique de la bobine, ..., qui leur communiquent une vitesse de diffusion dans le gaz. Par ailleurs, le gaz lui-même est animé de mouvements de convection dus à la dissipation thermique engendrée par le chauffage du filament d'évaporation. La vitesse moyenne d'une particule est donc égale à la somme de sa vitesse de diffusion $u$ dans le gaz et de la vitesse moyenne $\bar{U}$ du filet dans lequel elle est en suspension.

Les particules étant thermalisées dans le gaz, puisqu'elles sont capables de contourner des écrans optiquement étanches (cf. Fig. 1), la vitesse $u$ peut être calculée en utilisant la théorie cinétique et les lois de diffusion dans les gaz raréfiés [14]. Compte tenu des conditions expérimentales utilisées, on arrive à :

$$
10^{-4} \mathrm{~cm} \cdot \mathrm{s}^{-1} \leqslant u \leqslant 1 \mathrm{~cm} \cdot \mathrm{s}^{-1} .
$$

La valeur de la vitesse $\bar{U}$ peut être estimée en étudiant la nature de l'écoulement du gaz dans les condi- tions expérimentales utilisées. D'une part, la valeur du nombre de Grashof [15] montre que la convection est turbulente, d'autre part, la valeur du nombre de Reynolds [15] associée aux mouvements ascendant et descendant du gaz conduit à :

$$
\bar{U} \simeq 1500 \mathrm{~cm} \cdot \mathrm{s}^{-1}
$$

La comparaison des valeurs respectives des vitesses $u$ et $\bar{U}$ montre que les particules sont transportées de la zone où elles sont nucléées au voisinage de la surface de l'échantillon par les courants de convection turbulents du gaz.

La vitesse $\bar{U}$ n'est cependant pas la vitesse des particules au voisinage de la surface de l'échantillon. En effet, à cause de l'existence d'une couche limite, la vitesse $v_{0}$ est notablement inférieure à $\bar{U}$. Du fait de la structure des dispositifs expérimentaux décrits cidessus et des conditions expérimentales (libre parcours moyen des atomes du gaz de l'ordre de $10 \mu \mathrm{m}$ ), l'écoulement dans la couche limite est laminaire [14] et la vitesse $v_{0}$ est proportionnelle à $\bar{U}$ [14]. Elle peut être estimée à :

$$
v_{0} \simeq 60 \mathrm{~cm} \cdot \mathrm{s}^{-1}
$$

3. Performances des dispositifs existants. - La description précédente et l'estimation des ordres de grandeur des paramètres de la relation (1) permettent de discuter les performances des dispositifs existants : valeur de l'induction maximale $B_{\max }$ et probabilité de réussite.

La valeur de l'induction maximale à laquelle il est possible d'effectuer des décorations peut être estimée à 2000 gauss environ ce qui est cohérent avec les résultats expérimentaux obtenus jusqu'à présent.

La faible probabilité de réussite est liée au mode de transport des particules entre la zone où elles sont nucléées et la surface de l'échantillon. En effet, le caractère non déterminé et fluctuant du régime turbulent qui se développe à la suite de l'évaporation a deux incidences néfastes :

- La quantité de particules arrivant à un endroit donné de l'échantillon peut être trop importante ou trop faible.

- La vitesse d'écoulement $U$ peut atteindre localement des valeurs sensiblement plus élevées que la vitesse moyenne $\bar{U}$. Il en est de même pour $v_{0}$ et la valeur de l'induction maximale $B_{\max }$ peut s'en trouver grandement affectée.

Ainsi, une valeur de $v_{0}$ trois fois plus élevée que celle calculée plus haut conduit à :

$$
B_{\max } \lesssim 10 \text { gauss } .
$$

4. Nouvelle technique. - La discussion du paragraphe précédent montre qu'une amélioration notable du procédé de décoration peut être obtenue en s'affranchissant du caractère aléatoire du transport des 
particules. Ceci peut être obtenu en utilisant une technique qui permette :

- d'assurer le transport des particules par un écoulement laminaire du gaz,

- de contrôler la vitesse des particules.

Dans ce but, nous avons conçu et expérimenté un dispositif devant permettre de remplir ces deux conditions. Ce dispositif est constitué d'une chambre d'évaporation (cf. dispositifs précédents) qui se trouve en communication avec un conduit où est placé l'échantillon à décorer et où circule de l'hélium gazeux (Fig. 4). Ce conduit est limité par deux plans parallèles (l'un étant la surface de l'échantillon) dont la distance $h$ doit être telle que la turbulence engendrée par l'évaporation du fer ne puisse s'y propager. Pour ce faire,

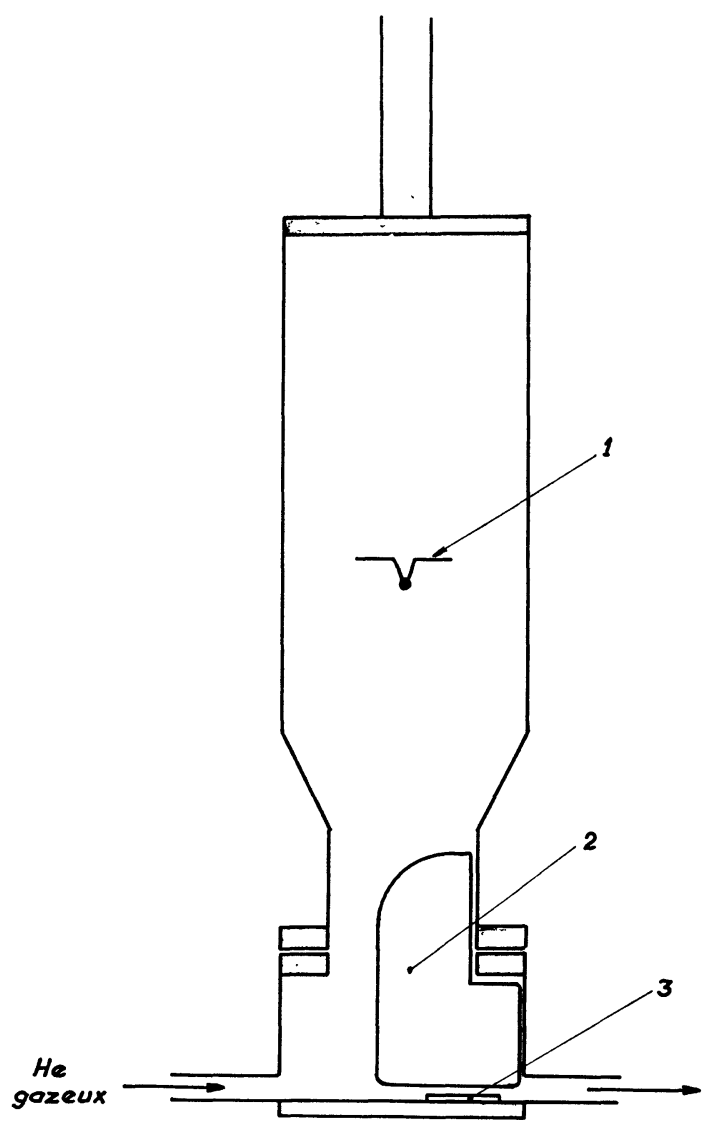

FIg. 4. - Nouveau dispositif : 1) filament d'évaporation ; 2) bloc de cuivre ; 3) échantillon.

$h$ doit être inférieure à la dimension du plus petit tourbillon existant dans cette turbulence. Cette dimension $\lambda_{0}$, appelée échelle interne de la turbulence [15], est, compte tenu des dimensions du récipient et $\mathrm{du}$ nombre de Reynolds, de l'ordre de $1 \mathrm{~mm}$. Afin de déterminer les meilleures conditions expérimentales, la distance $h$ pouvait être réglée entre 0,1 et $4 \mathrm{~mm}$.

Les premières expériences effectuées avec ce dispositif ont montré le caractère critique de la distance $h$ qui doit bien être du même ordre de grandeur que $\lambda_{0}$. En effet lorsque $h$ est supérieure à $1 \mathrm{~mm}$ environ, la probabilité de réussite reste voisine de $10 \%$ (cf. dispositifs précédents) mais elle atteint $70 \%$ environ lorsque cette distance est inférieure ou égale à $1 \mathrm{~mm}$ (cf. Fig. 5). Ces premières expériences ont été effectuées sur des échantillons supraconducteurs dont l'induction était d'une centaine de gauss ; il n'a pas encore été possible de travailler à des inductions plus élevées car le dispositif ne permet pas actuellement d'assurer un contrôle suffisant de la taille et de la vitesse des particules.

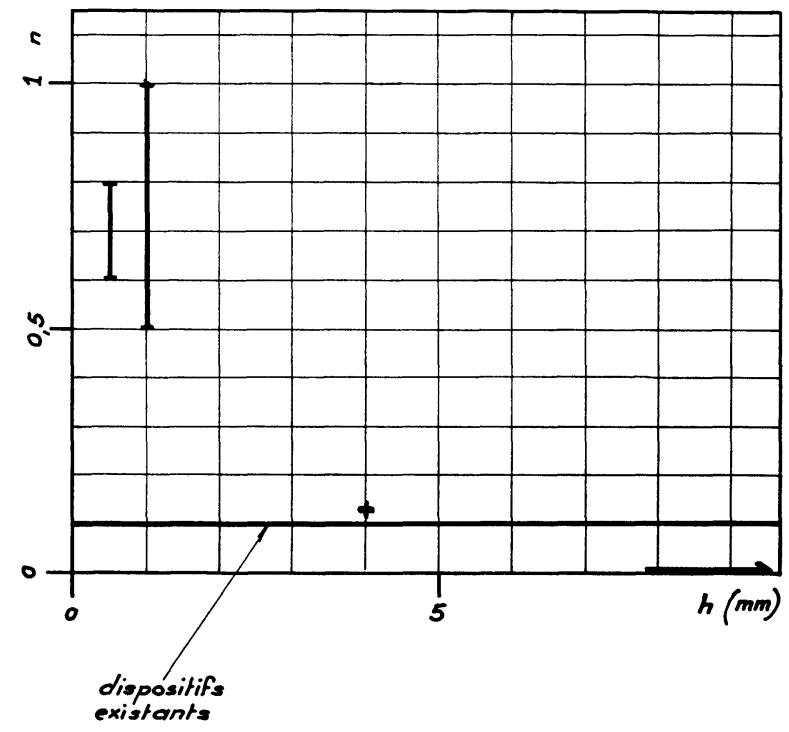

FIG. 5. - Probabilité de réussite $n$ du nouveau dispositif en fonction de la hauteur $h$ du conduit d'écoulement. Les barres d'erreur tiennent compte des échantillons qu'il n'a pas été possible d'examiner au microscope électronique à cause de la réplique.

5. Conclusion. - L'étude des processus qui interviennent pendant la décoration des réseaux de vortex avec des particules magnétiques, nous a permis de mettre en évidence l'origine des limitations des performances des dispositifs utilisés jusqu'à présent : transport aléatoire des particules. Ceci nous a conduit à concevoir une nouvelle technique permettant de contrôler l'arrivée des particules au voisinage de l'échantillon à décorer. Les résultats des premières expériences effectuées avec un dispositif mettant en œuvre cette technique ont confirmé, en grande partie, le bien-fondé de notre analyse et nous avons pu faire passer la probabilité de succès de $10 \%$ à $70 \%$. Ces travaux seront poursuivis afin de pouvoir travailler à des inductions plus élevées.

Remerciements. - L'auteur tient à remercier M. J. C. Renard et M. G. Pendaries, des Laboratoires de Marcoussis, qui ont participé à cette étude ainsi que M. le Professeur Y. Simon, M. P. Thorel et J. M. Delrieu pour de fructueuses discussions. 


\section{Bibliographie}

[1] Bitter, F., Phys. Rev. 38 (1931) 1903.

[2] Trauble, H., Essmann, U., Phys. Lett. 24A (1967) 526.

[3] Trauble, H., Essmann, U., Die direkte Beobachtung einzelner Linien magnetischen Flusses in Supraleitern (Jahrbuch der Akademie der Wissenschaften in Gottingen), 1967.

[4] Trauble, H., Ussmann, U., J. Appl. Phys. 39 (1968) 4052.

[5] Essmann, U., Phys. Lett. 41A (1972) 477.

[6] Sarma, N. V., Moon, J. R., Phil. Mag. 16 (1967) 433.

[7] Sarma, N. V., Moon, J. R., Phil. Mag. 17 (1967) 501.

[8] Sarma, N. V., Phil. Mag. 18 (1968) 1233.

[9] Sarma, N. V., Phil. Mag. 18 (1968) 171.
[10] Sarma, N. V., Wilcokson, A., Phil. Mag. 20 (1969) 539.

[11] BRANDT, E. H., Calculation of magnetization curves and microscopic functions of the triangular and square vortex lattices near $T_{\mathrm{c}}$ for all values of $\kappa$ and $B$. Discussion meeting on structure of mixed state in type II superconductors, Jülich, April 1972.

[12] Milleron, P. F., Fournet, G., Franzinetit, M., J. low Temp. Phys. 4 (1971) 545.

[13] Tasaki, A., TomiYama, S., IIDA, S., Wada, N., Uyeda, R., Jap. J. Appl. Phys. 4 (1964) 707.

[14] KenNARD, Kinetic theory of gases (ed McGraw Hill), 1938.

[15] LANDAU et LifCHITZ, Mécanique des fluides (ed. Mir), 1971. 